\title{
Extracorporeal Membrane Oxygenation in Hyperthyroidism-Related Cardiomyopathy: Two Case Reports
}

\author{
Vincent Pong ${ }^{\mathrm{a}}$, Chun-Yip Yeung ${ }^{\mathrm{b}}$, Ryan Lap-Yan $\mathrm{Ko}^{\mathrm{a}}$, \\ Hung-Fat Tse ${ }^{a}$, Chung-Wah Siu ${ }^{\text {a, }}$
}

\begin{abstract}
Hyperthyroidism-related cardiomyopathy complicated by acute heart failure and shock is a life-threatening condition. Due to the co-occurrence of systemic congestion, left ventricular systolic dysfunction, and reduced systemic vascular resistance, clinical management remains a major challenge in the intensive care unit. Extracorporeal membrane oxygenation (ECMO) provides temporary support in patients with refractory heart failure and cardiogenic shock. We describe two patients with severe hyperthyroidism-related cardiomyopathy. The first patient, a 33-year-old Chinese male, was newly diagnosed to have hyperthyroidism, presenting with thyroid storm and multi-organ failure. The second patient, a 35-yearold Chinese female, had relapsed Graves' disease complicated with hyperthyroidism-related cardiomyopathy and atrial fibrillation. Both patients developed acute heart failure and circulatory collapse, necessitating invasive hemodynamic support. They were hemodynamically supported with veno-arterial mode extracorporeal membrane oxygenation (VA-ECMO) and mechanical ventilation, which were successfully weaned off after 4 days. Subsequent echocardiography and cardiac magnetic resonance revealed normalization of the left ventricular ejection function. Hyperthyroidism-related cardiomyopathy with circulatory collapse is often a fatal condition. Given its highly reversible nature, more aggressive initial mechanical circulatory support in the form of VA-ECMO is warranted.
\end{abstract}

Keywords: Hyperthyroidism; Cardiomyopathy; Extracorporeal membrane oxygenation

Manuscript accepted for publication January 31, 2013

${ }^{a}$ Division of Cardiology, Department of Medicine, Li Ka Shing Faculty of Medicine, the University of Hong Kong, Hong Kong, China

${ }^{b}$ Division of Endocrinology and Metabolism, Department of Medicine,

Li Ka Shing Faculty of Medicine, the University of Hong Kong, Hong

Kong, China

${ }^{\mathrm{c} C o r r e s p o n d i n g ~ a u t h o r: ~ C h u n g ~ W a h ~ S i u, ~ D i v i s i o n ~ o f ~ C a r d i o l o g y, ~}$ Department of Medicine, Li Ka Shing Faculty of Medicine, The University of Hong Kong, Queen Mary Hospital, Hong Kong, China. Email: cwdsiu@hku.hk

doi: http://dx.doi.org/10.4021/jem144w

\section{Introduction}

Hyperthyroidism is a common metabolic disorder with prominent cardiovascular manifestations. In a non-selected cohort of hyperthyroid patients we have previously reported that while $5.8 \%$ of patients developed symptoms of congestive heart failure, less than half of these $(2.7 \%)$ had dilated cardiomyopathy with left ventricular systolic dysfunction [1]. Although it is generally accepted that hyperthyroidismrelated cardiomyopathy is reversible following achievement of a euthyroid state, a minority of patients in the course of hyperthyroidism may develop fatal circulatory collapse in addition to congestive heart failure. Due to the co-occurrence of systemic congestion, left ventricular systolic dysfunction, reduced systemic vascular resistance, and cardiac tachyarrhythmia, acute hemodynamic management remains a major challenge in the intensive care unit. Extracorporeal membrane oxygenation (ECMO) has been widely used to mechanically support the heart and lungs in patients with severe heart failure to recovery or to transplantation. This report documents two cases of near-fatal hyperthyroidismrelated cardiomyopathy with congestive heart failure and circulatory collapse successfully managed with veno-arterial (V-A) mode ECMO.

\section{Case Report}

\section{Case 1}

A 33-year-old Chinese male enjoying good health was admitted to a local hospital with a one week history of progressive shortness of breath and palpitations. A few months earlier he had noted heat intolerance, weight loss, and diarrhea. On admission, his temperature was $39.2^{\circ} \mathrm{C}$; blood pressure was $90 / 48 \mathrm{mmHg}$ with irregular pulse at rate 170 beats per minute; respiratory rate was 32 breaths per minutes. Physical examination revealed an elevated jugular venous pressure, bilateral ankle edema, and bilateral basal crackles over his chest. There were no murmurs detected. Chest X-ray showed cardiomegaly and acute pulmonary edema. Electrocardio- 


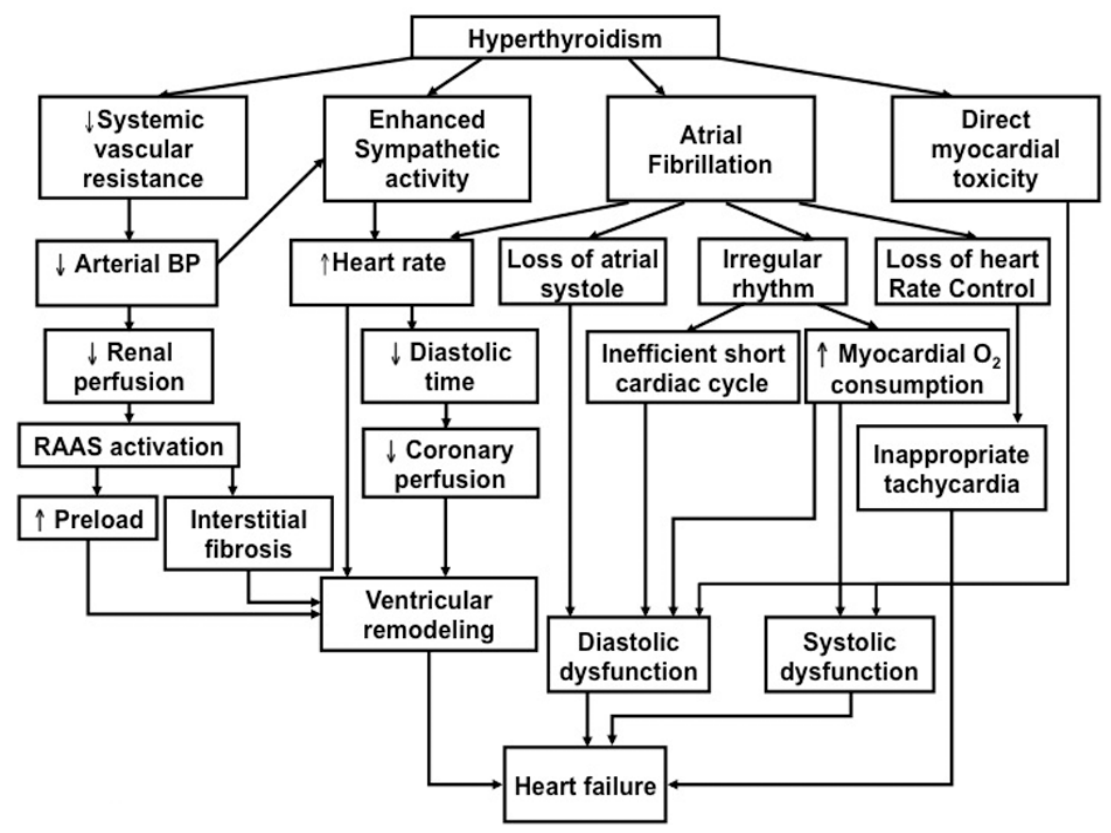

Figure 1. Schematic diagram depicting possible mechanisms underlying the patho-physiology of hyperthyroidism-related heart failure.

gram (ECG) showed fast atrial fibrillation (AF) of 170 beats per minute with no acute ischemic changes. Clinically, he was in acute congestive heart failure and cardiogenic shock. He was intubated, commenced on inotropic support with dopamine and dobutamine, and transferred to the intensive care unit.

His condition continued to deteriorate with profound cardiogenic shock and systolic blood pressure $<70 \mathrm{mmHg}$ despite inotropic support. Laboratory investigations revealed evidence of multi-organ failure including acute liver failure, acute renal failure and disseminated intravascular coagulopathy. Specifically, there were elevated levels of transaminases (alanine transaminase $>3,000$ units/L (normal range 42 - 110 units/L) and aspartate transaminase $>3,000$ units $/ \mathrm{L}$ (normal range: 15 - 38 units/L)), an elevated total bilirubin of $108 \mu \mathrm{mol} / \mathrm{L}$ (normal range: 4 - $23 \mu \mathrm{mol} / \mathrm{L}$ ), and a prolonged prothrombin time of 46 seconds (normal range: $11.3-13.5$ seconds). In addition, serum creatinine level rose to 361 $\mu \mathrm{mol} / \mathrm{L}$ (normal range: $67-109 \mu \mathrm{mol} / \mathrm{L}$ ) with serum urea level of 14.8 (normal range: $3.2-8.0 \mathrm{mmol} / \mathrm{L}$ ). Ultrasonography of the abdomen did not reveal any hepatic or renal pathology. Transthoracic echocardiogram showed grossly dilated left and right ventricles with severely impaired left ventricular systolic function and ejection fraction of $10 \%$. There were no significant valvular lesions. Because of the hemodynamic collapse, he was put on VA-ECMO. On the second admission day, a diagnosis of hyperthyroidism was confirmed with an elevated level of free thyroxine $\left(\mathrm{T}_{4}\right)$ of $55 \mathrm{pmol} / \mathrm{L}$ (normal range: 12 - $22 \mathrm{pmol} / \mathrm{L}$ ) and a suppressed thyroid-stimulating hormone (TSH). The Burch-Wartofsky score was 100 , highly suggestive of thyroid storm. He was thus treated with propranolol, propylthiouracil, Lugol's iodine and intravenous hydrocortisone. His hemodynamics gradually stabilized and he was successfully weaned off VAECMO 4 days later with subsequent improvement in liver and kidney biochemistry. The free $\mathrm{T}_{4}$ level was normalized at 1 week. At 2 weeks, heart failure was clinically resolved and spontaneous sinus conversion was noted. He was prescribed carvedilol and perindopril for left ventricular systolic dysfunction. Cardiac magnetic resonance imaging 3 weeks after discharge from the intensive care unit revealed only mild residual left ventricular systolic dysfunction with LVEF of $51 \%$ and dilated left ventricle with end-diastolic volume. There was no evidence of myocardial ischemia or scar.

\section{Case 2}

A 35-year-old Chinese female was admitted to the emergency department with a 1 week history of progressive shortness of breath on exertion and bilateral ankle swelling. She complained also of nausea and diarrhea on the day of admission but had no fever, chest pain, or palpitations. Her history was significant for Graves' disease diagnosed 7 years ago, treated with an 18-month course of anti-thyroid drug. Physical examination confirmed tachypnea with a respiratory rate of 25 breaths per minute. Blood pressure was $155 / 83$ and pulse rate was fast and irregular. Bilateral pitting ankle edema, raised jugular venous pressure and basal crackles over both lung fields were also noted. There was a moderate goiter with thyroid bruit. Chest X-ray revealed cardiomegaly and 
pulmonary edema. ECG showed fast atrial fibrillation (166/ $\mathrm{min}$ ) with no ischemic change. Laboratory evaluation revealed normal creatinine kinase and troponin I level but free thyroxine $\mathrm{T}_{4}$ was elevated ( $44 \mathrm{pmol} / \mathrm{L}$ ) with a TSH. Transthoracic echocardiogram revealed grossly dilated left and right ventricles with LVEF of $17 \%$. The Burch-Wartofsky score was 65 suggesting a high possibility of thyroid storm; oral propranolol, propylthiouracil, Lugol's iodine and intravenous hydrocortisone were started. As she remained in fast atrial fibrillation with ventricular rate $>150$ beats per minute and with oxygen desaturation requiring $100 \%$ oxygen supplement, digoxin and diltiazem for ventricular rate control, and frusemide for congestion were given. Eight hours later she developed profound cardiogenic shock with blood pressure $75 / 53 \mathrm{mmHg}$ and type II respiratory failure. Intubation with mechanical ventilation supported with VA-ECMO was initiated and her condition was stabilized. VA-ECMO was removed 4 days later with normalization of the serum free $\mathrm{T}_{4}$ level. She was clinically out of heart failure with spontaneous sinus conversion. In view of the impaired left ventricular function, ramipril was commenced. Propylthiouracil was gradually tapered as radioactive iodine treatment was planned as definitive management of her Graves' disease. Two months later, cardiac magnetic resonance imaging showed partial recovery of LVEF to $52 \%$ with no evidence of myocardial ischemia or fibrosis.

\section{Discussion}

Heart failure associated with hypotension is one of the most catastrophic complications of hyperthyroidism with mortality as high as $30 \%$ [2]. Thyroid hormone exerts diverse effects on the cardiovascular system and can aggravate pre-existing cardiac disease or cause de novo cardiovascular abnormalities [1, 3-5]. Figure 1 illustrates various mechanisms through which a hyperthyroid state leads to clinical heart failure. The hallmark hemodynamic change in hyperthyroidism is the marked reduction in systemic vascular resistance, and thus systemic arterial blood pressure $[4,5]$. This can in turn activate the renin-angiotensin-aldosterone system to increase preload and total blood volume by promoting salt and fluid retention, as well as the activation of the sympathetic nervous system to increase heart rate and heart force. The net result can be translated into an increased cardiac output for up to $250 \%$ at resting state. This nonetheless limits the body's ability to further increase cardiac output by increasing the heart rate, ejection fraction, and/or lowering systemic vascular resistance. The ability of the functional cardiac reserve to cope with the demand during exercise or other stresses is thus reduced. Of note, evidence from experimental work [6] as well as clinical observations [7] demonstrated that thyroid hormone exerts direct effects on cardiomyocytes that can enhance their mechanical function. Nevertheless it may also cause myocardial damage, eventually culminating in the development of systolic [6] and diastolic dysfunction [8]. Lastly, atrial fibrillation as well as its associated hemodynamic effects (loss of atrial systole and atrio-ventricular synchrony, irregular ventricular activation, and loss of heart rate control) is often the final trigger for cardiac decompensation in hyperthyroidism: while only $5-15 \%$ of patients develop AF during the course of hyperthyroidism [1, 3, 9-13], it is invariably present in patients with hyperthyroidism-related heart failure [1].

The goals of management of hyperthyroid heart failure and hypotension are (1) to restore a euthyroid state and (2) to maintain adequate tissue perfusion and oxygenation. The former usually involves a multimodal treatment approach including beta-adrenergic blockade, antithyroid drug therapy, inorganic iodide, and corticosteroid therapy to reduce the synthesis, release, and conversion of bioactive thyroid hormone [14]. Unfortunately the regimen often requires hours to days to be effective. In the meantime, effective measures to maintain tissue perfusion and oxygenation are of paramount importance. Although the final consequence of salt and water retention is similar to the more commonly encountered low output heart failure, the treatment options for hyperthyroidism-related heart failure differ significantly. Unlike heart failure in other settings that is associated with high peripheral vascular resistance, hyperthyroidism-related heart failure is associated with reduced peripheral vascular resistance. As a result, the use of diuretic and/or vasodilator therapy in the acute phase despite clinical hypervolemia may aggravate the hemodynamic instability. As in the second case, rapid hemodynamic deterioration developed shortly following administration of diltiazem for ventricular rate control of AF. Although diltiazem is a more effective alternative in ventricular rate control in hemodynamically stable fast AF of non-thyroid etiology [15], its prominent vasodilatory effects may aggravate the pathophysiology. The use of propranolol, a non-selective beta-blocker, as part of anti-thyroid therapy [16], has also a theoretical advantage of blocking both $\beta_{1}$ adrenergic receptors on the heart to slow down ventricular rate for $\mathrm{AF}$ and $\beta_{2}$-receptors at peripheral circulation to reduce vasodilatation. There have nonetheless been reported cases of patients with thyroid storm who developed cardiac arrest shortly after the initiation of propranolol [17]. As such, use of ultra-short acting beta-blockers, such as intravenous esmolol, has been suggested with extreme caution. Current guidelines recommend direct current cardioversion for $\mathrm{AF}$ in hemodynamically unstable patients although in the presence of hyperthyroidism the AF often persists [18]. Repeated attempts at direct current cardioversion may result in myocardial stunning which further jeopardize the cardiac output.

ECMO is a modified form of cardiopulmonary bypass that maintains tissue oxygenation from days to weeks in critically ill patients with respiratory and/or heart failure [19]. In VA ECMO, a.k.a. cardiac ECMO, deoxygenated blood is 
drawn, via a large cannula in the vena cava by a pump, to pass through an artificial lung for oxygenation and removal of carbon dioxide. Oxygenated blood is then returned to the arterial circulation via a peripheral cannula, typically in the femoral artery. ECMO is commonly indicated in those with compromised hemodynamic status as a bridging therapy to heart transplantation, but also occasionally as a bridging therapy to allow cardiovascular recovery. Concurrent with previous studies and case reports, upon restoration of a euthyroid state, rapid normalization of hemodynamics, spontaneous termination of AF, and improvement in left ventricular systolic function were observed in both of our cases, suggesting the highly reversible nature of hyperthyroidismrelated cardiomyopathy. More aggressive initial circulatory support in the form of VA-ECMO should therefore be considered earlier in the course of hyperthyroidism-related cardiomyopathy with circulatory collapse.

\section{Conclusion}

Hyperthyroidism-related cardiomyopathy with circulatory collapse is often a fatal condition. Given its highly reversible nature, more aggressive initial mechanical circulatory support in the form of VA-ECMO is warranted.

\section{Competing Interest}

The authors declare that they have no competing interests.

\section{Authors' Contributions}

VP reviewed and summarized patients' data. VP and CY were major contributors in writing the manuscript. All authors read and approved the final manuscript.

\section{References}

1. Siu CW, Yeung CY, Lau CP, Kung AW, Tse HF. Incidence, clinical characteristics and outcome of congestive heart failure as the initial presentation in patients with primary hyperthyroidism. Heart. 2007;93(4):483487.

2. Nayak B, Burman K. Thyrotoxicosis and thyroid storm. Endocrinol Metab Clin North Am. 2006;35(4):663-686, vii.

3. Siu CW, Pong V, Zhang X, Chan YH, Jim MH, Liu S, Yiu KH, et al. Risk of ischemic stroke after new-onset atrial fibrillation in patients with hyperthyroidism. Heart Rhythm. 2009;6(2):169-173.

4. Siu CW, Zhang XH, Yung C, Kung AW, Lau CP, Tse HF. Hemodynamic changes in hyperthyroidism-related pul- monary hypertension: a prospective echocardiographic study. J Clin Endocrinol Metab. 2007;92(5):1736-1742.

5. Wong SM, Tse HF, Siu CW. Pulmonary hypertension and isolated right heart failure complicating amiodarone induced hyperthyroidism. Heart Lung Circ. 2012;21(3):163-165.

6. Lee YK, Ng KM, Chan YC, Lai WH, Au KW, Ho CY, Wong LY, et al. Triiodothyronine promotes cardiac differentiation and maturation of embryonic stem cells via the classical genomic pathway. Mol Endocrinol. 2010;24(9):1728-1736.

7. Marti V, Ballester M, Obrador D, Moya C, Carrio I, Pons-Llado G. Active myocardial damage in hyperthyroidism. A concurrent mechanism of heart failure reversed by treatment. Eur Heart J. 1995;16(7):10141016.

8. Yue WS, Chong BH, Zhang XH, Liao SY, Jim MH, Kung AW, Tse HF, et al. Hyperthyroidism-induced left ventricular diastolic dysfunction: implication in hyperthyroidism-related heart failure. Clin Endocrinol (Oxf). 2011;74(5):636-643.

9. Siu CW, Jim MH, Zhang X, Chan YH, Pong V, Kwok J, Kung AW, et al. Comparison of atrial fibrillation recurrence rates after successful electrical cardioversion in patients with hyperthyroidism-induced versus non-hyperthyroidism-induced persistent atrial fibrillation. Am J Cardiol. 2009;103(4):540-543.

10. Kerr CR, Boone J, Connolly SJ, Dorian P, Green M, Klein G, Newman D, et al. The Canadian Registry of Atrial Fibrillation: a noninterventional follow-up of patients after the first diagnosis of atrial fibrillation. Am J Cardiol. 1998;82(8A):82N-85N.

11. Sawin CT, Geller A, Wolf PA, Belanger AJ, Baker E, Bacharach P, Wilson PW, et al. Low serum thyrotropin concentrations as a risk factor for atrial fibrillation in older persons. N Engl J Med. 1994;331(19):1249-1252.

12. Woeber KA. Thyrotoxicosis and the heart. N Engl J Med. 1992;327(2):94-98.

13. Polikar R, Burger AG, Scherrer U, Nicod P. The thyroid and the heart. Circulation. 1993;87(5):1435-1441.

14. Bahn RS, Burch HB, Cooper DS, Garber JR, Greenlee $\mathrm{MC}$, Klein I, Laurberg P, et al. Hyperthyroidism and other causes of thyrotoxicosis: management guidelines of the American Thyroid Association and American Association of Clinical Endocrinologists. Endocr Pract. 2011;17(3):456-520.

15. Siu CW, Lau CP, Lee WL, Lam KF, Tse HF. Intravenous diltiazem is superior to intravenous amiodarone or digoxin for achieving ventricular rate control in patients with acute uncomplicated atrial fibrillation. Crit Care Med. 2009;37(7):2174-2179; quiz 2180.

16. Riaz K, Forker AD, Isley WL, Hamburg MS, McCullough PA. Hyperthyroidism: a "curable" cause of congestive heart failure--three case reports and a review 
of the literature. Congest Heart Fail. 2003;9(1):40-46.

17. Dalan R, Leow MK. Cardiovascular collapse associated with beta blockade in thyroid storm. Exp Clin Endocrinol Diabetes. 2007;115(6):392-396.

18. Nakazawa HK, Sakurai K, Hamada N, Momotani N, Ito
K. Management of atrial fibrillation in the post-thyrotoxic state. Am J Med. 1982;72(6):903-906.

19. Gaffney AM, Wildhirt SM, Griffin MJ, Annich GM, Radomski MW. Extracorporeal life support. BMJ. 2010;341:c5317. 